\title{
Manifestations
}

6 - 9 Juillet 2008

\section{ESHRE 24th Annual Meeting}

Barcelone, Espagne

Information : www.eshre.com

3 - 8 Août 2008

Gordon Research Conferences: Reproductive Tract Biology

Andover, NH, USA

Information : www.grc.org/meetings

19 - 21 Septembre 2008

8th Panhellenic Congress of Andrology

Athens, Greece

Information : www.andrologyGR2008.com

10 - 11 Octobre 2008

XXVème Congrès de la SALF

Tunisie

Information : www.salf.fr

8 - 12 Novembre 2008

ASRM Annual Meeting (American Society for Reproductive Medicine)

San Francisco, CA, USA

Information : www.asrm.org

26 - 28 Novembre 2008

European Academy of Andrology: Vth ECA Congress

Rome, Italy

Information : www.andrology2008.com 